\title{
The problem of $\mathrm{C}_{7}^{-}$as source of diffuse interstellar bands revisited
}

\author{
G. Galazutdinov ${ }^{1,2}$, F. Musaev ${ }^{1,2}$, J. Nirski ${ }^{2}$, and J. Krełowski ${ }^{2}$ \\ 1 Special Astrophysical Observatory, Nizhnij Arkhyz 369167, Russia \\ e-mail: faig@sao.ru \\ 2 Center for Astronomy, Nicholas Copernicus University, Gagarina 11, 87-100 Toruń, Poland \\ e-mail: nirski@astri.uni.torun.pl, jacek@astri.uni.torun.pl
}

Received 22 March 2001 / Accepted 8 August 2001

\begin{abstract}
We used spectra of several bright, reddened stars, most of them likely to be obscured by individual interstellar clouds, to examine the proposition that $\mathrm{C}_{7}^{-}$may carry several diffuse interstellar bands (DIBs) (Tulej et al. 1998). To search for the broad features of the $\mathrm{C}_{7}^{-} \mathrm{B} \leftarrow \mathrm{X}$ system we used low resolution, very high $S / N$ spectra, but even in heavily reddened objects the predicted bands remain undetectable. High resolution $(R=80000)$ spectra prove that the diffuse bands, found in close vicinity of narrow lines belonging to the $\mathrm{A} \leftarrow \mathrm{X}$ system, show variable strength ratios from object to object which proves that they are not of the same origin. The paper brings thus evidence that the $\mathrm{C}_{7}^{-}$molecule does not cause any of the detected DIBs.
\end{abstract}

Key words. ISM: clouds, molecules

\section{Introduction}

The problem of identification of diffuse interstellar bands (DIBs) is the longest standing unsolved problem in all of spectroscopy (Herbig 1995). Recently, the most often presented hypotheses connect these puzzling spectral structures with different kinds of complicated interstellar molecules, usually some carbon-bearing species (for a review see Fulara \& Krełowski 2000). Among the proposed species these, based on linear carbon skeletons, are the most popular ones. Bare carbon chains have been proposed as DIB carriers many years ago (Douglas 1977) but until very recently their spectra have not been known from laboratory gas-phase experiments. The existing, matrixisolation spectra do not allow a direct comparison because of broadening and possible wavelength shifts caused by the crystals of noble gases. Interstellar clouds produce spectral features most likely in conditions of very rariefied gas. This is why gas-phase laboratory spectra are essential to test any hypothesis of DIB origin.

Recently Tulej et al. (1998) used the technique of resonance-enhanced two-colour photodetachment to obtain sharp gas-phase spectra of carbon-chain anions. They have indicated this anion as possible source of five DIBs tabulated by Jenniskens \& Désert (1994). This possible identification was discussed by

Send offprint requests to: G. Galazutdinov e-mail: gala@sao.ru
Galazutdinov et al. (1999) who concluded that $\mathrm{C}_{7}^{-}$carbon chains do not cause any of the detectable diffuse interstellar bands. They emphasized a necessity of high resolution echelle spectroscopy which allows to determine wavelengths of unidentified interstellar features with a high precison, using radial velocities measured in profiles of interstellar atomic lines recorded in the same spectra. Also the importance of using stars of different velocities of rotation was stressed as an important test allowing to distinguish between weak stellar and interstellar spectral features. Another important point is to compare spectra of different individual clouds. As indicated many years ago (Krełowski \& Westerlund 1988) intensity ratios of different DIBs may seriously differ from cloud to cloud. Spectral features can be considered as a spectrum of a single species only if their strength ratios are the same along every line of sight. Thus any proposed set of DIBs must be observed towards several stars in order to make sure they are of the same origin. Let's emphasize that any case of evidently different strength ratio makes the identification at least doubtful.

McCall et al. (2000) presented another analysis of the same possible spectral features. They have observed four heavily reddened stars to test the $\mathrm{C}_{7}^{-}$hypothesis. A high reddening should make a detection of any interstellar feature easier but also makes impossible a determination of effects taking place in individual clouds. Any heavily reddened star is observed through several clouds along the 
sightline. Their optical properties are very likely to be different and the observed spectrum is an ill-defined average of all these clouds. With a sufficiently high number of clouds along a sightline the observed spectra are very likely to be more or less the same towards different stars because they converge to the same average. The choice of objects, made by McCall et al. (2000) makes impossible to test behaviour of interstellar features in individual spectra i.e. to check whether their intensity ratios are the same in spectra of different individual clouds.

The stars chosen by McCall et al. (2000) are all supergiants of relatively late spectral types. This precludes a possibility to distinguish between stellar and interstellar features basing on differing rotational velocities or different spectral types of the selected stars. Supergiants always rotate very slowly; spectra of late B stars are full of weak stellar lines. Moreover sightlines towards heavily reddened stars typically intersect clouds differing in radial velocities (as it has been convincingly shown in the case of HD 183143 by Hebig \& Soderblom, 1982) and thus profiles of interstellar features cannot resemble those obtained in laboratories. A reliable comparison of profiles obtained in laboratory and observations is thus not possible in cases of heavily reddened targets (see also Fig. 8 of McCall et al.).

The aim of the present paper is to re-analyze the problem of possible identification of $\mathrm{C}_{7}^{-}$features in spectra of reddened stars. The analyses, mentioned as necessary in the former paper (Galazutdinov et al. 1999), have been conducted here i.e. we used high resolution spectra of stars characterized by different spectral types, rotational velocities and different strength ratios of major diffuse bands. They permitted an analysis of the strength ratios of the narrow lines of the $\mathrm{A} \leftarrow \mathrm{X}$ system, reliably separated from stellar or telluric contaminations. We searched for the broad $\mathrm{B} \leftarrow \mathrm{X}$ lines in low resolution spectra which allow more likely to trace these spectral lines.

\section{The observational data}

The low resolution spectra have been acquired with the aid of the Torun Observatory $0.9 \mathrm{~m}$ telescope equipped with the Richardson spectrometer. This spectrometer was set to record spectra with the resolution of 3000, covering the range between 4300 and $5300 \AA$. Very high $S / N$ ratio (1000) makes even very weak features detectable. The expected $\mathrm{B} \leftarrow \mathrm{X}$ lines should be as broad as several tens of angstroems (Tulej et al. 1998); they should be detected in our spectra if their EW's are above $0.05 \AA$. High resolution spectra, especially if acquired with an echelle spectrometer, are not useful to detect broad features of the width comparable to those of single echelle orders. In such cases our low resolution spectra allow a reliable continuum setting even over very broad features. The targets observed in low resolution are listed in Table 1; they differ in spectral types and rotational velocities but a special emphasizis is put on very hot objects containing only a few lines in their spectra. The another important factor is the broad range of reddenings inside our sample.
Table 1. The list of observed stars. Top half are objects observed in low resolution, bottom - these in high resolution. First column - HD number; second - spectral and luminosity class; third - reddening; fourth - rotational velocity; fifth (high res only) - number of averaged spectra.

\begin{tabular}{rlrrr}
\hline HD & SpL & $E_{B-V}$ & $v \sin i$ & $N$ \\
\hline 47839 & O7Ve & 0.04 & 65 & \\
24534 & O9.5pe & 0.56 & 120 & \\
164353 & B5Ib & 0.1 & 60 & \\
24912 & O7e & 0.31 & 216 & \\
207198 & O9IIb & 0.54 & 80 & \\
190603 & B1.5Iae & 0.7 & 32 & \\
\hline 23180 & B1III & 0.27 & 85 & 1 \\
24398 & B1Iab & 0.29 & 60 & 6 \\
179406 & B3V & 0.31 & 150 & 2 \\
206165 & B2Ib & 0.46 & 35 & 1 \\
207198 & O9IIb & 0.54 & 80 & 1 \\
210839 & O6Iab & 0.52 & 300 & 4 \\
\hline
\end{tabular}

We decided also to use the spectra of resolution 80000 acquired with the aid of the coudé echelle spectrometer (Musaev et al. 1999) fed by the $2 \mathrm{~m}$ telescope of the Observatory on top of the peak Terskol (Northern Caucasia) to analyze the spectral features of $\mathrm{A} \leftarrow \mathrm{X}$ system which are narrow. High resolution allows profile analysis of these features as they contain not less than 20 points inside every profile. The stars observed with the high resolution (twice as large as that of McCall et al.) are listed in Table 1. The targets were chosen using the profiles of atomic interstellar lines. In all the chosen lines of sight the Doppler splitting is barely seen in very narrow atomic lines. Profiles of DIBs are much broader and thus we may consider them as free of Doppler effect. Such a choice of targets allows not only to measure intensities of the chosen bands but also to analyze their profiles.

The spectrometer, working with the Wright Instruments CCD $1242 \times 1152$ matrix (pixel size $22.5 \mu \mathrm{m} \times 22.5 \mu \mathrm{m}$ ) camera, covers in two exposures the range $\sim 3500 \AA-\sim 10100 \AA$ with the resolution $R=80000$. Any exposure covers approximately one half of the above mentioned range.

Our reduction of the echelle spectra was made using the DECH code (Galazutdinov 1992). This program allows flatfield division, bias/background subtraction, onedimensional spectrum extraction from the 2-dimensional images, correction for the diffuse light, spectrum addition, excision of cosmic ray features, etc. The DECH code also allows location of a fiducial continuum, measurements of the line equivalent widths, line positions and shifts, etc. The spectral range, covered in every exposure, contains strong and well-identified atomic interstellar lines: CaII, CaI, NaI and KI. This allowed us to determine precisely radial velocities of the intervening interstellar clouds at a moment of any observation.

The determined radial velocities allowed us to shift the whole spectra to the rest wavelength scale for interstellar features. Such a procedure, is developed in the DECH 


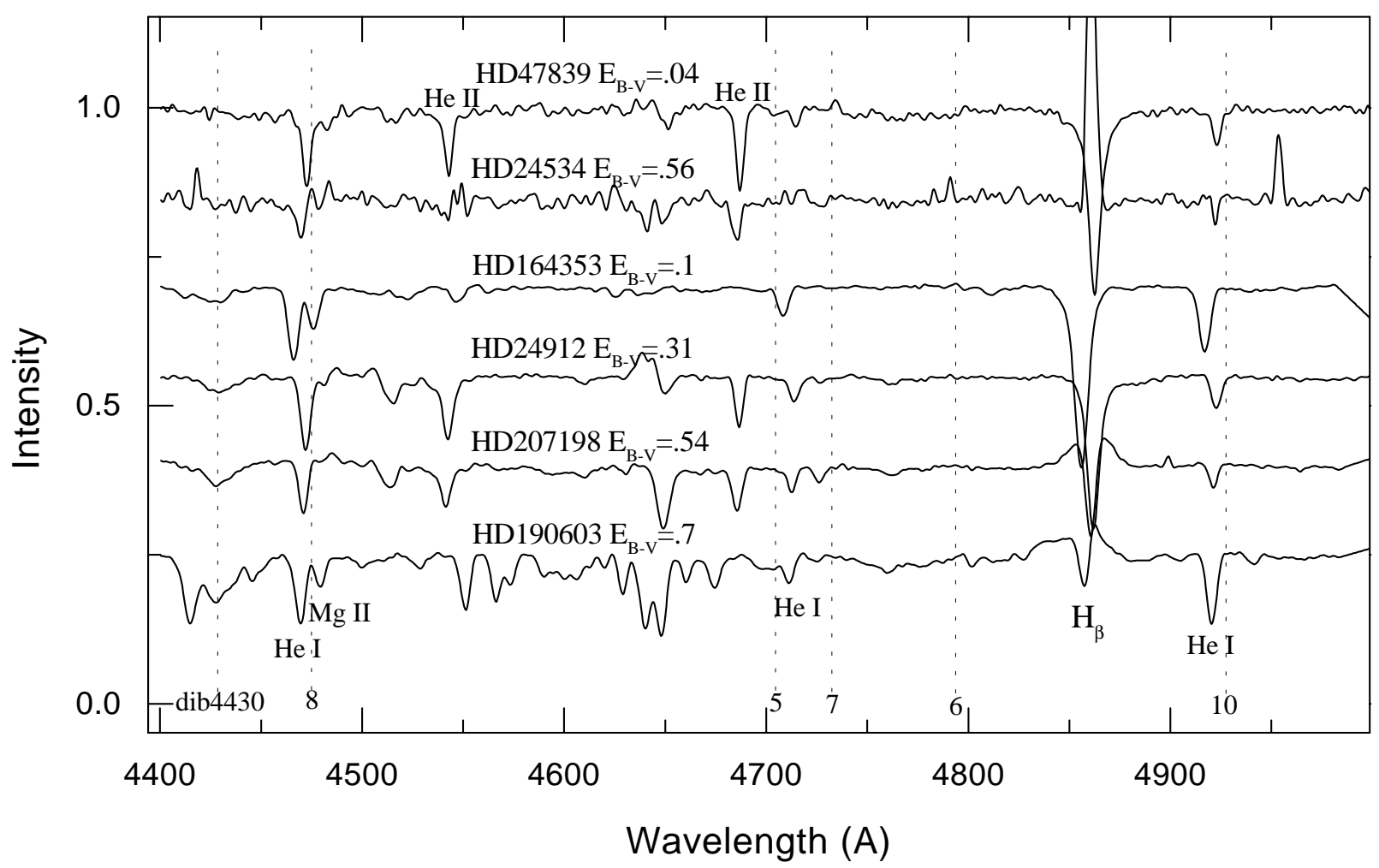

Fig. 1. Low resolution spectra from the Torun spectrometer, covering the range of the $\mathrm{B} \leftarrow \mathrm{X}$ system of $\mathrm{C}_{7}^{-}$molecule. The positions of the possible features are marked and their expected relative intensities - given. Note the clear presence of the well-known $4430 \AA$ DIB in several spectra.

code: wavelength of each spectrum point is corrected to the value corresponding to the same radial velocity. A set of spectra shifted to the rest wavelength scale for the interstellar intervening clouds allow to search for weak interstellar features by means of averaging spectra of different targets. While adding the spectra after the radial velocity correction, the interstellar features are "amplified" i.e. they become visible even while not detectable in individual spectra (Krełowski \& Schmidt 1997).

This procedure is especially useful while trying to determine strength ratios of strong and weak features. The latter are barely detectable in individual spectra as our targets are not heavily reddened. The averaging procedure resembles the observations of heavily reddened stars where also many clouds along one sightline create an average with one important difference: in heavily reddened targets the features are Doppler-splitted. We avoid this effect, correcting every spectrum to the rest wavelength velocity scale of the intervening clouds. Thus the averaged high resolution profiles can be compared to those determined in laboratory. The only physical importance of an averaged spectrum is the possibility of determination of intensity ratios of analyzed features if we try to check whether these ratios resemble closely those predicted in laboratory eperiments. If all the features listed by Tulej et al. (1998) originate in the $\mathrm{C}_{7}^{-}$molecule, then their intensity ratios should be the same in every individual spectrum and thus - also in the averaged, high resolution and very high $S / N$ spectrum.
Table 1 characterizes our sample of target stars, i.e. their HD numbers, spectral types, luminosity classes, colour excesses and rotational velocities. It also shows the number of spectra of each star used to produce final averaged spectra covering the $\mathrm{C}_{7}^{-}$features. The reddened targets have been observed together with some standards and divisors (HD 120315, HD 116658, HD 218045) to separate the interstellar features from the stellar and telluric contaminations.

All the chosen targets are reasonably bright stars which was essential as the applied high resolution makes the achievement of high $S / N$ quite difficult. Typical $S / N$ ratio in individual spectra was $\sim 300$ and we found it not sufficiently high to measure the weak $\mathrm{C}_{7}^{-}$features.

\section{Results}

The low resolution spectra of the targets listed in Table 1 are depicted in Fig. 1. We have also marked the positions of the expected broad spectral structures of the $\mathrm{B} \leftarrow \mathrm{X}$ band of $\mathrm{C}_{7}^{-}$. The expected bands are clearly undetectable despite the quality of spectra i.e. their possible EW's are below $0.05 \AA$. The well-known, broad DIB $4430 \AA$ is clearly seen, even in the spectrum of HD 164353 used as the unreddened standard by McCall et al. Some of the proposed spectral lines are very likely to be blended with strong stellar lines but these which are not close to any stellar line are also not visible. Apparently the set of 


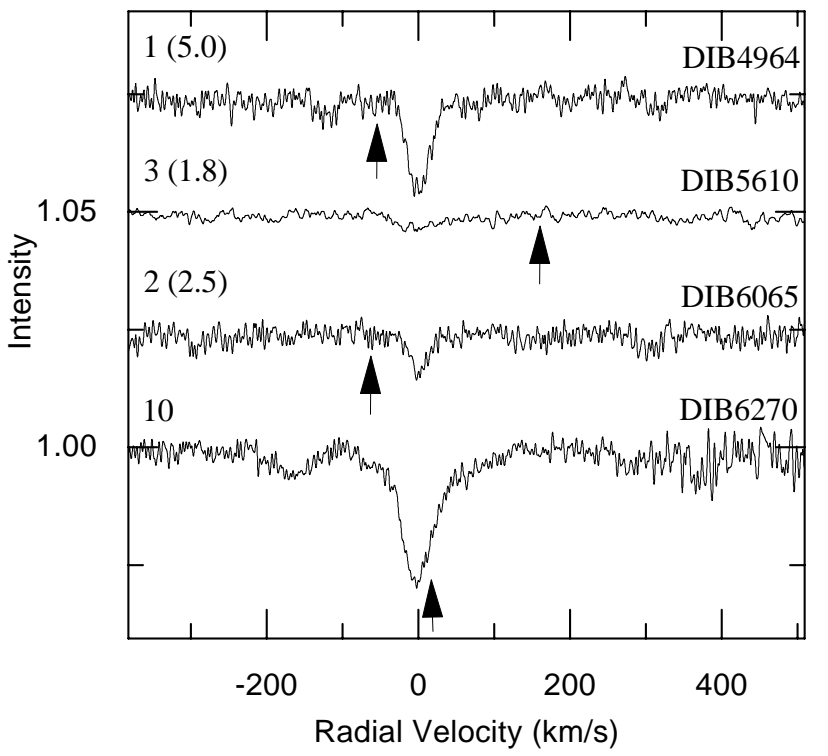

Fig. 2. The averaged, high resolution profiles of the four analyzed DIBs. The positions of $\mathrm{C}_{7}^{-} \mathrm{A} \leftarrow \mathrm{X}$ system lines are marked with arrows. The laboratory relative intensities are given as well as those - measured (in parentheses). The latter differ strongly from the laboratory expectations.

spectra from Table 1 does not support the idea that the spectrum of $\mathrm{C}_{7}^{-}$participates in interstellar absorptions.

We have averaged the high resolution, echelle spectra of the objects listed in Table 1 which allowed to create the spectrum of resolution $R=80000$ and $S / N \sim 1000$. The resultant, averaged spectral ranges around the suspect features are shown in Fig. 2 where the radial velocity scale was applied to compare the bands in one frame. Only four features out of eight found in laboratory by Tulej et al. can be traced in our spectra - the same which have been observed by McCall et al. (2000). Our comparison shows also the laboratory positions of the expected lines. They are up to $3 \AA$ shifted from the closest interstellar bands.

The most important result is the fact that the measured intensity ratios of the interstellar features differ seriously from those found in the laboratory. The $4964 \AA$ DIB which should be the weakest one, an order of magnitude weaker than the main $6270 \AA \mathrm{DIB}$, is only two times weaker. The band around $5610 \AA$ which should be the second strongest is the weakest etc. The strength ratios of the interstellar fetaures do not resemble those published by Tulej et al. as well as those, slightly corrected by McCall et al. It does not mean that the measurements of McCall et al. are not correct. They concern different targets and thus they only confirm the variability of the strength ratios. This variability is a strong argument against any common origin of the analyzed DIBs.

The variable ratios of the analyzed DIBs are depicted in Fig. 3. It shows the two DIBs of the intensity ratio most closely resembling that predicted in laboratory experiments, 6270 and $6065 \AA$ observed in spectra of two reasonably reddened, bright stars: HD 210839 and HD 207198.

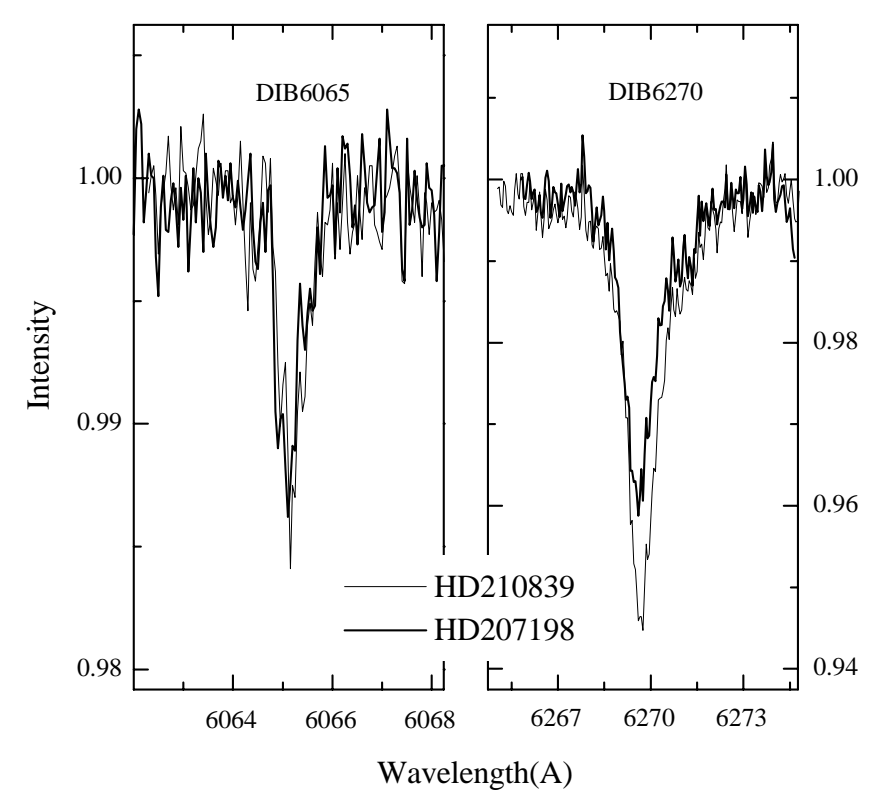

Fig. 3. The observed variable ratio of two analyzed features seen in the spectra of two different reddened stars.

The ratio is apparently different, so even these two features are very likely to be of different origin.

Two of our targets were observed many times (HD 24398 and HD 210839). This gives us a possibility to compare their averaged spectra being of reasonable $S / N$ ratio. The result is shown in Fig. 4. The intensity ratio of the two strongest DIBs, depicted also in Fig. 2 i.e. $4964 \AA$ and $6270 \AA$ is evidently variable. The range of variability is similar to that, found by Krełowski \& Westerlund (1988) in the case of $5780 \AA$ and $5797 \AA$ major DIBs. This fact leaves little doubt that the two depicted features are of different origin, so they cannot both belong to a spectrum of any single molecule, including of course, $\mathrm{C}_{7}^{-}$.

We have not made a plot, similar to any of those, depicted in Figs. 3 and 4 presenting the feature near $5610 \AA$ because the feature is very weak (contrary to laboratory predictions) which makes such a comparison of intensity ratios not reliable.

We should also comment the question whether all the analyzed features are of interstellar origin. First argument follows Fig. 2. The observed targets characterized by different spectral types and rotational velocities have been averaged. Stellar lines are in such cases very likely to disappear. Only the interstellar bands can get amplified while spectra shifted to the interstellar rest wavelength velocity scale are averaged. A second argument follows Fig. 3. The depicted targets are of seriously different rotational velocities. Despite of this the profiles of considered features are identical which strongly supports their interstellar origin.

Our results clearly show that only analysing spectra of moderately reddened stars, which are likely to be observed through single interstellar clouds, one can check whether any proposed set of DIBs can be of common origin which is a necessary step towards an identification. Very high $S / N$ 


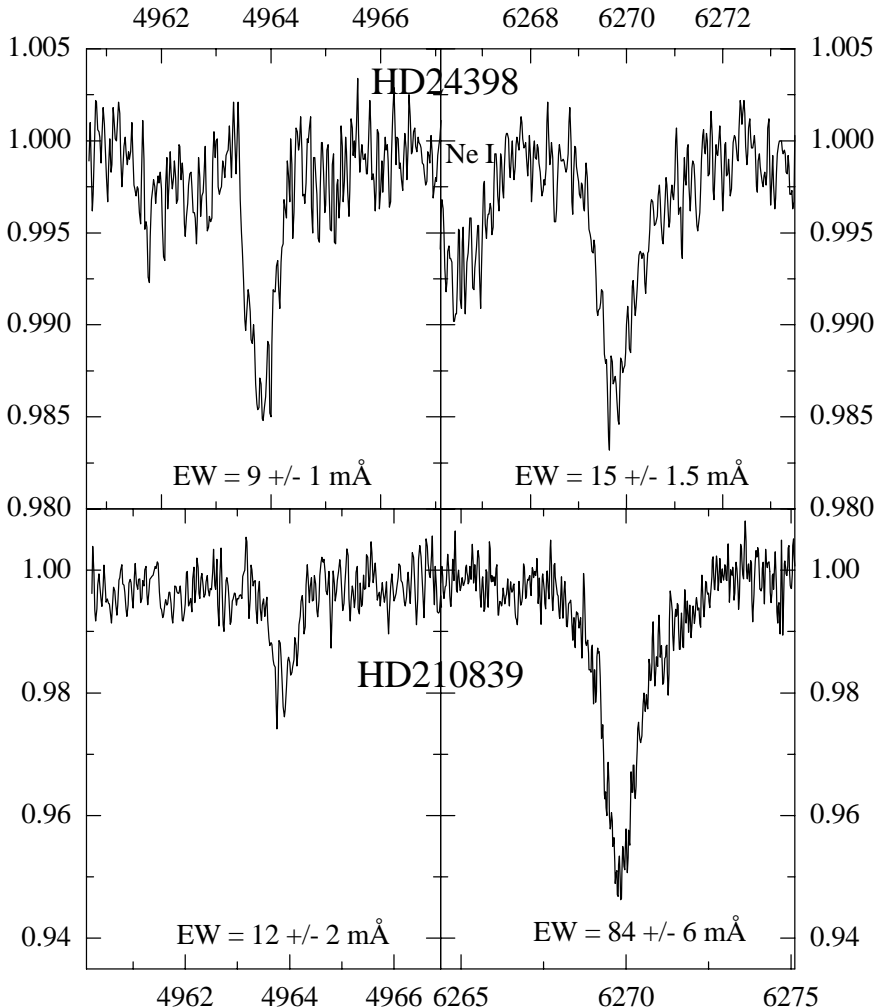

Fig. 4. Two of the observed DIBs seen in spectra of two reddened stars attributed to the $\mathrm{A} \leftarrow \mathrm{X}$ system of $\mathrm{C}_{7}^{-}$by Tulej et al. (1998). Note the strongly variable strength ratio.

ratio is crucial because any interstellar feature is weak when observed in slightly reddened stellar spectra.

\section{Discussion}

The analysis presented in this paper is a decisive argument against the identification of $\mathrm{C}_{7}^{-}$molecule as a source of several DIBs. The only support of this hypothesis comes from the proximity of four lines from the $A \leftarrow X$ system of this molecule to four evidently interstellar features. However, we found several strong arguments against this possibility:

- intensity ratios of the observed DIBs (related by Tulej et al. to the $\mathrm{A} \leftarrow \mathrm{X}$ system of the $\mathrm{C}_{7}^{-}$molecule) differ from object to object which contradicts the hypothesis of their common origin;

- the strength ratios of the observed DIBs differ strongly from those predicted (for the $\mathrm{A} \leftarrow \mathrm{X}$ system) in laboratory experiments; the differences cannot be explained as results of observational inaccuracies;

- the wavelengths of the observed features do not match exactly those predicted (for the $\mathrm{A} \leftarrow \mathrm{X}$ system) in laboratory experiments;

- the profile of the strongest $\mathrm{A} \leftarrow \mathrm{X}$ feature, near $6270 \AA$, does not resemble that of the observed DIB as already pointed out by Sarre \& Kendall (2000);

- none of the $\mathrm{B} \leftarrow \mathrm{X}$ system features of $\mathrm{C}_{7}^{-}$was detected despite rather high reddening of the targets observed and high $S / N$ ratio of the recorded spectra.

The observations, presented above provide a clear evidence that the observed DIBs, related by Tulej et al. (1998) to the $\mathrm{C}_{7}^{-}$molecule originate in some other species and their identification still requires additional experimental data. The molecule $\mathrm{C}_{7}^{-}$, if present in the interstellar clouds, is much less abundant than estimated assuming its spectral features are strong and easily observable.

Acknowledgements. The authors want to express their gratitude to the staff of the Terskol and Torun Observatories for the technical help during the observations. Our collaboration was supported by the NATO travel grant PST.CLG.976080. GAG wants to express his thanks to the NATO; the project was completed during his stay in Torun as a NATO advanced fellow.

\section{References}

Douglas, A. E. 1977, Nature, 269, 130

Fulara, J., \& Krełowski, J. 2000, New Astr. Rev., 44, 581 Galazutdinov, G. A. 1992, Preprint Spets. Astrof. Obs., 92

Galazutdinov, G. A., Krełowski, J., \& Musaev, F. A. 1999, MNRAS, 310, 1017

Galazutdinov, G. A., Musaev, F. A., Krełowski, J., \& Walker, G. A. H. 2000, PASP, 112, 648

Herbig, G. H., \& Soderblom, D. R. 1982, ApJ, 252, 610

Herbig, G. H., 1995, Ann. Rev. Astron. Astrophys., 33, 19

Jenniskens, P., \& Désert, F.-X. 1994, A\&AS, 106, 39

Krełowski, J., \& Westerlund, B. E. 1988, AAP, 190, 339

Krełowski, J., \& Schmidt, M. 1997, ApJ, 477, 209

McCall, B. J., York, D. G., \& Oka, T. 2000, ApJ, 531, 329

Musaev, F. A., Galazutdinov, G. A., Sergeev, A. V., Karpov, N. V., \& Podyachev Yu.V. 1999, Kinematika i Fizika Nebesnyh Tel, V. 15, No. 3

Sarre, P. J., Kendall, T. R. 2000, in Astrochemistry IAU Symp., 197, ed. Y. C. Minh, \& E. F. van Dishoeck (Kluwer), 343

Tulej, M., Kirkwood, D. A., Pachkov, M., \& Maier, J. P. 1998, ApJ, 506, L69 